TITLE:

\title{
Absorption enhancement of an electric quadrupole transition of cesium atoms in an evanescent field
}

\author{
$\operatorname{AUTHOR}(\mathrm{S}):$ \\ Tojo, S; Hasuo, M; Fujimoto, T
}

\section{CITATION:}

Tojo, S ... [et al]. Absorption enhancement of an electric quadrupole transition of cesium atoms in an evanescent field. Physical Review Letters 2004, 92(5): 053001.

\section{ISSUE DATE:}

2004-02-06

URL:

http://hdl.handle.net/2433/39922

RIGHT:

Copyright 2004 American Physical Society 


\title{
Recoil-Free Spectroscopy of Neutral Sr Atoms in the Lamb-Dicke Regime
}

\author{
Tetsuya Ido ${ }^{1, *}$ and Hidetoshi Katori ${ }^{1,2, \dagger}$ \\ ${ }^{1}$ Cooperative Excitation Project, ERATO, Japan Science and Technology Corporation, \\ 4-1-8 Hon-cho, Kawaguchi, Saitama 332-0012, Japan \\ ${ }^{2}$ Engineering Research Institute, The University of Tokyo, Bunkyo-ku, Tokyo 113-8656, Japan
}

(Received 1 March 2003; published 29 July 2003)

\begin{abstract}
Recoil-free as well as Doppler-free spectroscopy was demonstrated on the ${ }^{1} S_{0}-{ }^{3} P_{1}$ transition of $\mathrm{Sr}$ atoms confined in a one-dimensional optical lattice. By investigating the wavelength and polarization dependence of the ac Stark shift acting on the ${ }^{1} S_{0}$ and ${ }^{3} P_{1}\left(m_{J}=0\right)$ states, we determined the wavelength where the Stark shifts for both states coincide. This Stark-free optical lattice, allowing the purturbation-free spectroscopy of trapped atoms, may keep neutral-atom based optical standards competitive with single-ion standards.
\end{abstract}

DOI: 10.1103/PhysRevLett.91.053001

PACS numbers: $32.80 . P \mathrm{j}, 32.30 . \mathrm{Jc}, 32.60 .+\mathrm{i}, 32.70 . \mathrm{Jz}$

Recent dramatic advances in optical metrology have made it possible to directly link two optical frequencies with an uncertainty below $10^{-18}$ [1] and to coherently divide an optical frequency down to a radio frequency defined by the SI second [2]. Owing to these techniques, a stringent comparison of the stability and accuracy among optical clocks becomes feasible [3] and leads to an improved definition of time and tests of the time variation of fundamental constants [4]. Up to now, two sorts of absorbers, single ions in the Lamb-Dicke regime (LDR) [5] and neutral atoms in free space, have been extensively studied for optical clocks. Tightly confined single ions, enabling a long interaction time and the Doppler-free as well as the recoil-free absorption [6], have, so far, led to the narrowest optical spectrum [7]. However, the stability was severely limited by the quantum projection noise (QPN) [8] of the single absorber. The ensemble of neutral atoms, in contrast, provides a far better QPN limit. The accuracy of the measurement is known to be affected by the atomic motion, as residual Doppler shifts are introduced through the imperfect wave front of the probe beam $[9,10]$.

These two approaches could merge if an ensemble of neutral atoms was separately prepared in the LDR. An optical lattice made by the interference pattern of a light field [11] confines atoms in a small volume, which satisfies the Lamb-Dicke condition [5]. However, an impediment for the precision measurement is the complete control over the perturbations caused by this confinement, since the Stark shift potential strongly depends on the electronic state, which is not the case for iontrapping potentials.

The transition frequency between the states $|g\rangle$ and $|e\rangle$, subjected to the ac Stark shift of the trap laser with an electric field of $\vec{E}\left(\omega_{L}, \hat{\boldsymbol{\epsilon}}\right)$, can be written as

$$
\omega_{\text {obs }}=\omega_{0}-\Delta \alpha\left(\omega_{L}, \hat{\boldsymbol{\epsilon}}\right)\left|\vec{E}\left(\omega_{L}, \hat{\boldsymbol{\epsilon}}\right)\right|^{2} / 4 \hbar+O\left(\vec{E}^{4}\right) .
$$

Here, $\omega_{0}$ is the unperturbed atomic resonance frequency, $\hbar$ is the Planck constant, and

$$
\Delta \alpha\left(\omega_{L}, \hat{\boldsymbol{\epsilon}}\right)=\alpha_{e}\left(\omega_{L}, \hat{\boldsymbol{\epsilon}}\right)-\alpha_{g}\left(\omega_{L}, \hat{\boldsymbol{\epsilon}}\right)
$$

denotes the differential dipole polarizability. If the polarizabilities $\alpha_{e}$ and $\alpha_{g}$ coincide at a specific $\omega_{L}$ and $\hat{\boldsymbol{\epsilon}}$, the second term of Eq. (1) vanishes. Therefore, the unperturbed atomic transition frequency, independent of the trapping laser intensity of $I_{L} \propto|\vec{E}|^{2}$, can be observed $[12,13]$. This Stark shift cancellation technique was first demonstrated by improved atom loading into an optical trap for ultracold strontium atoms [12]. Similar ideas of tailoring ac Stark shift potentials have been employed for Raman sideband cooling [14] and fine spectroscopy [15] of alkali atoms in the lower-lying hyperfine states.

In this Letter, we report recoil-free spectroscopy of strontium atoms confined in a one-dimensional optical lattice by applying the technique to the $\left(5 s^{2}\right)^{1} S_{0}-$ $(5 s 5 p)^{3} P_{1}$ "clock" transition. By investigating the wavelength as well as the polarization dependence of the differential dipole polarizability, we have determined the wavelength, where the Stark shift on the clock transition vanishes, i.e., $\Delta \alpha=0$.

In order to discuss the polarization dependence of the Stark shift and the influence of Raman coherences between the $m, m^{\prime}$ magnetic sublevels of the $|a\rangle$ state, we introduced the light-shift Hamiltonian $V_{m^{\prime} m}$. We assume electric-dipole couplings to electronic states of $|b\rangle$ by fardetuned radiation with intensity $I_{L}$,

$$
V_{m^{\prime} m}=m \mu B \delta_{m^{\prime} m}-3 \pi c^{2} I_{L} \sum_{b} \frac{\gamma_{b a} \Lambda_{m^{\prime} m}(b, \hat{\boldsymbol{\epsilon}})}{\omega_{b a}^{2}\left(\omega_{b a}^{2}-\omega_{L}^{2}\right)} .
$$

The first term of Eq. (2) denotes the Zeeman energy in a magnetic field of magnitude $B$, where $\mu$ is the Zeeman shift coefficient of the state $|a\rangle$. The second term denotes the light shifts, where $\gamma_{b a}$ and $\omega_{b a}$ are the natural linewidth and the transition frequency for the $|b\rangle \rightarrow|a\rangle$ transition, respectively. The summation was carried out over the excited states, as described in our previous work [12]. $\Lambda(b, \hat{\boldsymbol{\epsilon}})$ denotes the coupling matrix element with a normalized dipole moment of $\hat{\mathbf{d}}_{b a}[16]$, 


$$
\Lambda_{m^{\prime} m}(b, \hat{\boldsymbol{\epsilon}})=\sum_{m_{b}}\left\langle m^{\prime}\left|\hat{\boldsymbol{\epsilon}} \cdot \hat{\mathbf{d}}_{b a}\right| m_{b}\right\rangle\left\langle m_{b}\left|\hat{\boldsymbol{\epsilon}}^{*} \cdot \hat{\mathbf{d}}_{b a}^{\dagger}\right| m\right\rangle,
$$

in which the summation runs over the magnetic sublevels $m_{b}$ in the $|b\rangle$ state accessed by the light polarization $\hat{\boldsymbol{\epsilon}}$. For the $a={ }^{1} S_{0}$ state, $\Lambda(b, \hat{\boldsymbol{\epsilon}})$ does not show any polarization dependence because of the isotropy of the state. However, for the upper state of the "clock transition" $a={ }^{3} P_{1}\left(m_{J}=0\right)$, the coupling strength $\Lambda(b, \hat{\epsilon})$ strongly depends on the light polarization. This is illustrated in Fig. 1(a) by the thickness of the arrows: taking the ${ }^{3} S_{1}$ state as the $|b\rangle$ state, this state is not coupled by $\pi$ polarized light but by $\sigma^{ \pm}$polarized light. In this way, the Stark shift for the ${ }^{3} P_{1}$ state is critically affected by the applied light polarization.

Our experimental setup and the procedure to form an optical lattice have been described elsewhere [17,18]. A few $\mu \mathrm{K}$ cold strontium atoms were magneto-optically cooled and trapped on the narrow ${ }^{1} S_{0}-{ }^{3} P_{1}$ transition [19]. During the final cooling and trapping period of $30 \mathrm{~ms}$, the atoms were loaded into a one-dimensional optical lattice. A frequency-stabilized Ti:sapphire laser (Coherent, MBR-110) was coupled into a polarizationmaintaining optical fiber and focused into the atom cloud by an objective lens. A quarter-wave and a half-wave plate were used to compensate the birefringence of the optical fiber and obtain arbitrary angular orientations $\theta$ of the laser polarization, as shown in Fig. 1(b). This trap beam, with a typical power of $500 \mathrm{~mW}$, was then retroreflected by a concave mirror with a radius of $25 \mathrm{~cm}$ to form a standing wave, where the $1 / e$ radius of the beam waist was $\approx 23 \mu \mathrm{m}$ at $\lambda_{L}=830 \mathrm{~nm}$.

With these parameters, the axial confinement frequency in the ${ }^{1} S_{0}$ state was measured to be $\nu_{S}=$

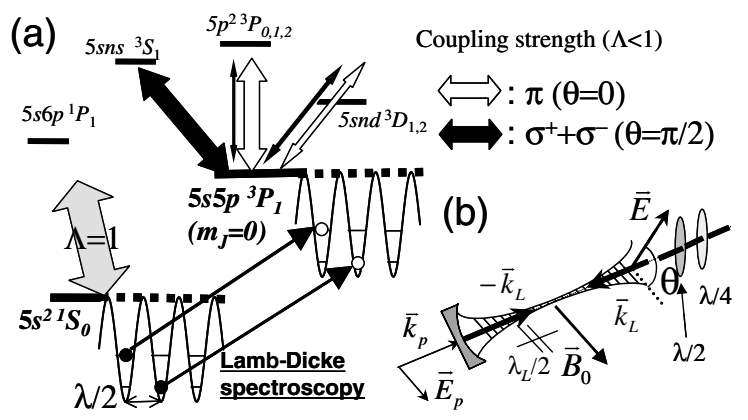

FIG. 1. (a) Level diagram and optical coupling related to the ${ }^{1} S_{0}-{ }^{3} P_{1}$ clock transition. The ${ }^{1} S_{0}$ state is dominantly coupled to ${ }^{1} P_{1}$, while the ${ }^{3} P_{1}$ state is coupled to the upper triplet states of ${ }^{3} S,{ }^{3} P$, and ${ }^{3} D$. The latter couplings strongly depend on the light polarization, as depicted by the thickness of arrows. (b) Schematic diagram of the 1D optical lattice. A bias magnetic field $\vec{B}_{0}$ was applied parallel to the probe electric field $\vec{E}_{p}$ with a wave vector $\vec{k}_{p}$ parallel to that of the lattice beam $\pm \vec{k}_{L} . \theta$ represents an angle between $\vec{B}_{0}$ and the electric field $\vec{E}$ of the lattice. The probe laser was introduced parallel to $\pm \vec{k}_{L}$, along which the spatial modulation of the ac Stark shift confines atoms in the LDR, as depicted in (a).
$90 \mathrm{kHz}$ by observing the first heating sideband, as discussed later. This vibrational frequency suggested a peak laser intensity of $I_{\text {peak }} \approx 47 \mathrm{~kW} / \mathrm{cm}^{2}$, which corresponds to a potential depth of $30 \mu \mathrm{K}$ and a radial trap frequency of $460 \mathrm{~Hz}$. Figure 1(b) shows a configuration for the spectroscopy. As the light source, we used an external cavity loaded laser diode electronically locked to a tunable reference cavity with its length stabilized to the $\mathrm{Sr}$ transition, thus achieving a laser linewidth of a few $\mathrm{kHz}$ over an integration time of half an hour. This probe beam was introduced through the dichroic concave mirror and superimposed on the lattice beam. The axial confinement of $\nu_{S}$ gave the Lamb-Dicke parameter $\eta=$ $\sqrt{h / 2 M \nu_{S}} / \lambda_{p}=0.24$, where $M$ is the atomic mass and $\lambda_{p}=689 \mathrm{~nm}$ is the wavelength of the ${ }^{1} S_{0}-{ }^{3} P_{1}$ clock transition. A bias magnetic field $\left|\vec{B}_{0}\right|=0.5 \mathrm{G}$ was applied parallel to the probe electric field $\vec{E}_{p}$ to define the quantization axis, which split the $m= \pm 1$ sublevels of the ${ }^{3} P_{1}$ state by $\pm \mu\left|\vec{B}_{0}\right| / h \approx \pm 1 \mathrm{MHz}$. The direction of the electric field $\vec{E}$ of the lattice laser was defined by an angle $\theta$ with respect to the quantization axis defined by $\overrightarrow{\boldsymbol{B}}_{0}$.

Figure 2(a) shows laser-induced fluorescence spectra for different electric field rotations $\theta \approx \pi / 4(A)$ and $\theta \approx$ $\pi / 2(B)$ at $\lambda_{L}=830 \mathrm{~nm}$. The probe intensity was $I_{p} \approx$ $3 I_{0}$ with $I_{0}=3 \mu \mathrm{W} / \mathrm{cm}^{2}$, the saturation intensity of the clock transition. The main peaks correspond to the $\left|{ }^{1} S_{0}, n_{v}\right\rangle \rightarrow\left|{ }^{3} P_{1}, n_{v}\right\rangle$ transition, where $n_{v}(=0,1,2 \cdots)$ represents the vibrational state of atoms in the Stark shift potential. The frequency difference of the axial confinement $\delta \nu=\nu_{P}-\nu_{S}$ for the ${ }^{3} P_{1}$ and ${ }^{1} S_{0}$ states caused a vibrational-state-dependent frequency shift of $n_{v} \delta \nu$ : The spectrum $(B)$ was inhomogeneously broadened as the several vibrational states were populated. In contrast, a simple picture of sideband cooling can be applied for the case of $A$, where vibrational frequencies are nearly degenerate. A small peak at $100 \mathrm{kHz}$ corresponded to excitation of the $\Delta n_{v}=+1$ heating sideband, while the $\Delta n_{v}=-1$ excitation (cooling sideband) was not visible as the atoms were quickly sideband cooled to the vibrational ground state [20].

The change of the resonance frequency is summarized in Fig. 2(b) as a function of the angular orientation $\theta$. Since the ${ }^{1} S_{0}$ state has no polarization dependence, the frequency change is attributed to the ac Stark shift of the ${ }^{3} P_{1}\left(m_{J}=0\right)$ state. The solid curve in Fig. 2(b) shows the differential Stark shift obtained from Eq. (2), and the dashed lines, the corresponding Stark shifts for the $\Delta n_{v}=0$ vibrational transitions with $n_{v}=0,1,2$. The vertical spread of these curves explains the broadening of the spectrum, as discussed previously. The observed frequency shift should be smaller than the $n_{v}=0$ differential Stark shift because the thermal distribution is typically $\langle n\rangle \approx 3$.7. Since the applied Zeeman shift of $\approx$ $1 \mathrm{MHz}$ was strong enough to suppress Raman coherence among Zeeman sublevels, the polarization dependence 

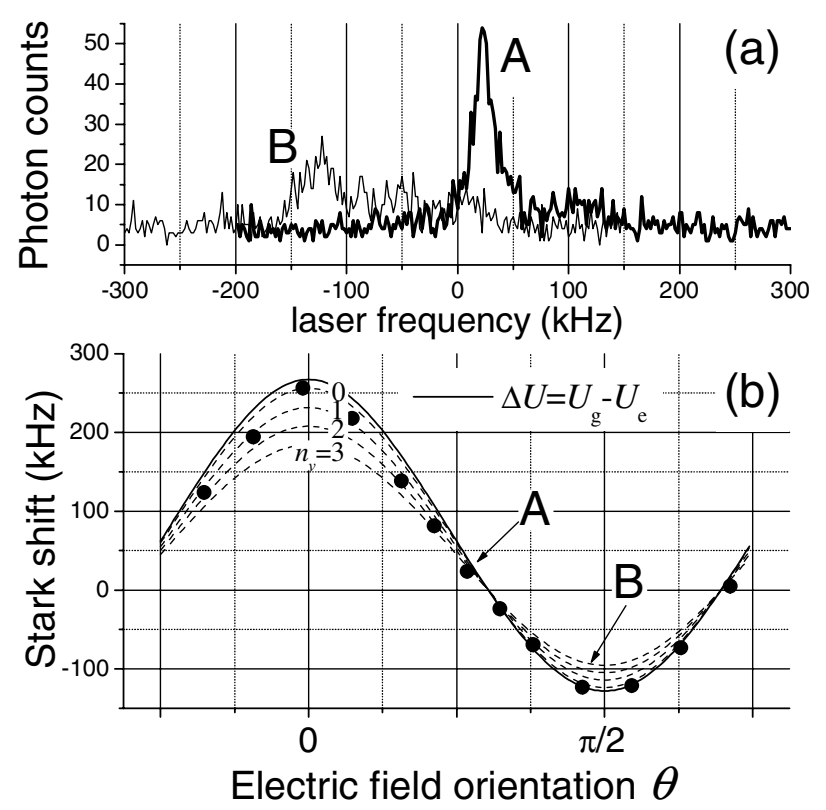

FIG. 2. (a) Fluorescence spectra of $\mathrm{Sr}$ atoms confined in a $1 \mathrm{D}$ optical lattice for different polarization orientation $\theta$ at $\lambda_{L}=$ $830 \mathrm{~nm}$. At $\theta \approx \pi / 4(A)$, the narrower spectrum was observed as the Stark shifts on the clock transition nearly canceled out, whereas nonzero differential Stark shift introduced a frequency shift and broadening for $\theta \approx \pi / 2(B)$. (b) The change of the Stark shift as the angular orientation $\theta$ of the latticelaser polarization with respect to a bias magnetic field. Since the light shift of the ${ }^{3} P_{1}\left(m_{J}=0\right)$ depends on the lattice laser polarization, the frequency varies sinusoidally depending on the components of $\pi$ and the $\sigma^{+}+\sigma^{-}$intensity. The dashed lines show the differential Stark shifts for atoms in the $n_{v}=$ $0,1,2$ vibrational states.

mainly accounted for the intensity of the $\pi$ polarized light $(\theta=0)$ and the linear combination of $\sigma^{+}+\sigma^{-}$ $(\theta=\pi / 2)$, resulting in a sinusoidal change. As the deviation from the theoretical curve was within $5 \%$, in the following analysis to determine the light shifts for $\theta=0$ and $\theta=\pi / 2$, we approximated the fitting curve by a sinusoidal function.

Figure 3 shows the Stark shifts for the $\theta=0$ and $\pi / 2$ in the range of $\lambda_{L}=810-930 \mathrm{~nm}$, where the Stark shift is normalized by the laser intensity $I_{\text {eff }} \approx 47 \mathrm{~kW} / \mathrm{cm}^{2}$. We note that, in the wavelength range $\lambda_{L}=(690)-915 \mathrm{~nm}$, in which the Stark shifts for the $\theta=0$ and $\pi / 2$ have an opposite sign, the differential polarizability $\Delta \alpha$ may vanish for a specific polarization orientation $\theta$, as demonstrated in Fig. 2(a). The observed efficient loading of atoms from the magneto-optical trap into the optical trap at $\lambda_{L} \sim 800 \mathrm{~nm}[12,17]$ can be attributed to this angletuning mechanism.

In order to accurately determine the unperturbed atomic resonance frequency $\omega_{0}$ free from Stark shifts, we measured the power dependence of the transition frequency at several $\lambda_{L}$ for $\theta=\pi / 2$, as shown in the inset in Fig. 3. By linearly extrapolating $I_{L} \rightarrow 0$, the measure-

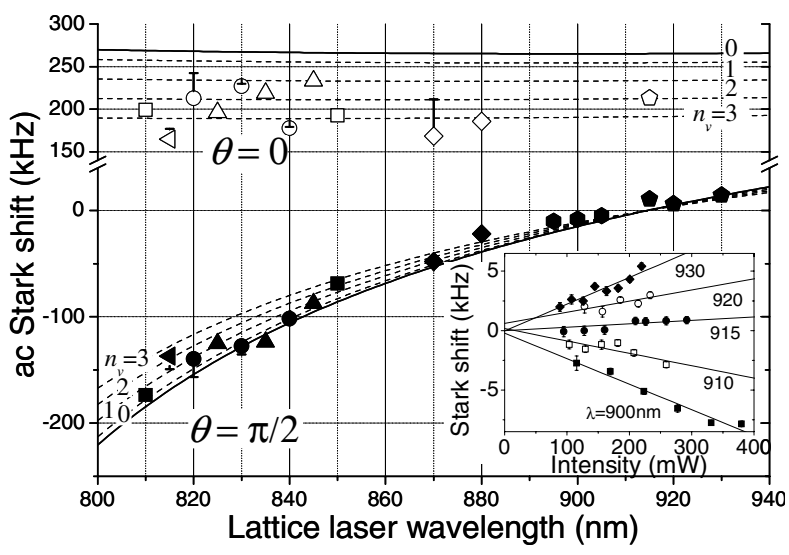

FIG. 3. Differential Stark shift for the ${ }^{1} S_{0}-{ }^{3} P_{1}(m=0)$ transition. The shift depends on the trap laser wavelength $\lambda_{L}$, the polarization rotation $\theta$, and the vibrational state $n_{v}$, indicated by dashed lines. Different symbols correspond to the measurements on different days. The inset shows the intensity dependence of the shifts measured for several wavelengths $\lambda_{L}$ at $\theta=\pi / 2$. By linearly extrapolating to zero laser power, the atomic resonance frequency was obtained.

ments at different wavelengths converged within $1 \mathrm{kHz}$, which was limited by the center frequency reproducibility of our laser system. Taking this value as a reference for the atomic resonance frequency, the wavelength which cancels the Stark shift was determined to be $\lambda_{L}=914 \pm$ $1 \mathrm{~nm}$ for $\theta=\pi / 2$. The inset also infers that the hyperpolarizability does not contribute in this range of intensities within the achieved measurement accuracy.

We used this wavelength to determine the uncertain transition moments in the calculation of the Stark shifts. Among the transition strengths listed in Ref. [21], the $(5 s 6 s)^{3} S \rightarrow(5 s 5 p)^{3} P$ transition rate showed a rather large uncertainty of more than $30 \%$. Therefore, we took this rate as a fitting parameter to zero the differential Stark shift at $\lambda_{L}=914 \mathrm{~nm}$. The estimated transition rate of $A=8.5 \times 10^{7} / \mathrm{s}$ was within the reported values of $6.7-9.2 \times 10^{7} / \mathrm{s}$ [21]. Using this value, we calculated the differential Stark shifts shown by the solid lines in Fig. 3. The difference between the data points and the theoretical curves is attributed to (i) the imperfect lattice laser polarization, as indicated by error bars in the figure, (ii) the uncertainty in determining the center frequency of the spectrum due to the vibrational state spread, as indicated by dashed lines, and (iii) the day-to-day change of the beam overlap of the lattice standing wave and resultant intensity fluctuations. The determination of the Stark-free wavelength, however, should be rather accurate, since this point is independent of laser intensity fluctuation and of vibrational states of the confined atoms.

Figure 4 demonstrates a recoil-free spectrum for $\theta=$ $\pi / 2$ at $\lambda_{L}=915 \mathrm{~nm}$, in which the Stark-free condition is nearly satisfied. The atom number was decreased to $\sim 10^{4}$, or $\sim 10$ atoms in each lattice site, to moderate the attenuation of the probe beam. We interrogated the atoms for 


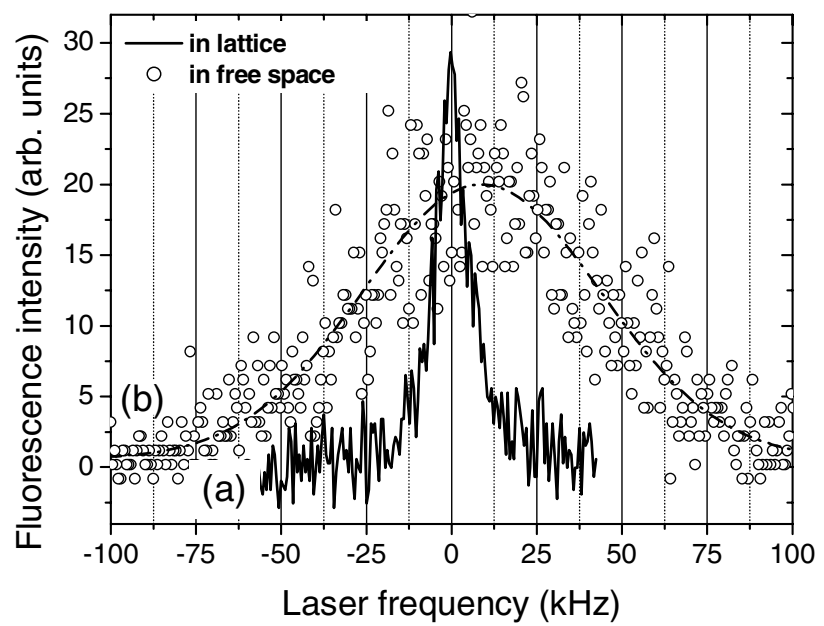

FIG. 4. Laser-induced fluorescence of atoms (a) confined in a 1D optical lattice and (b) in free fall. The dashed line shows a Gaussian fit to the data points (b). The confinement suppressed the Doppler width of $83 \mathrm{kHz}$ and gave a narrow Lorentzian linewidth of $11 \mathrm{kHz}$, which was limited by the saturation broadening. A slight blueshift of the center frequency in (b) is caused by the photon recoil shift.

$\tau_{p}=2 \mathrm{~ms}$ in each measurement and averaged 32 scans. The measured linewidth of $11 \mathrm{kHz}$ (FWHM) was in agreement with the saturation width for the probe intensity of $I_{p} \approx I_{0}$.

In order to demonstrate the modification of the atomic spectrum by the LDR, we measured the laser-induced fluorescence of free atoms. Figure 4(b) shows the fluorescence for a probe duration of $\tau_{p}=1.0 \mathrm{~ms}$, applied $1 \mathrm{~ms}$ after turning off the optical lattice. The Doppler width of $83 \mathrm{kHz}$ (FWHM) suggested the atom temperature of $6 \mu \mathrm{K}$ in the lattice. The slight blueshift of $\delta f=9 \mathrm{kHz}$ was attributed to the photon recoil shift as well as the recoil heating of atoms, since more than a single photon was scattered during the probe period $\tau_{p}$. By reducing the duration $\tau_{p}$ and extrapolating $\tau_{p} \rightarrow 0$, we obtained $\delta f\left(\tau_{p} \rightarrow 0\right)=5.2 \pm 0.6 \mathrm{kHz}$, in agreement with the recoil shift of $h /\left(2 M \lambda_{p}^{2}\right)=4.8 \mathrm{kHz}$.

In summary, we have demonstrated recoil-free spectroscopy of neutral ${ }^{88} \mathrm{Sr}$ atoms confined in the LambDicke regime provided by a $1 \mathrm{D}$ optical lattice. By adjusting the Stark shifts for the probed upper and lower states, we canceled out the perturbation of the lattice potential on the atom spectrum, thus realizing a system similar to ion-trapping experiments [6]. The application of this scheme to a frequency standard is promising because simultaneous preparation of $N$ neutral atoms improves the quantum projection noise by a factor of $\sqrt{N}$ compared to the single-ion-based standard [7]. The frequency accuracy of the present scheme may finally be limited by the purity of the lattice-laser polarization. The use of the ${ }^{1} S_{0}-{ }^{3} P_{0}$ transition of ${ }^{87} \mathrm{Sr}$ dramatically reduces polarization dependence [13] and thus may provide an alternative for the optical-frequency standard.
We thank M. Kuwata-Gonokami for valuable comments and support and Y. Li and T. Mukaiyama for their participation in discussions and assistance with the experiments.

*Current address: JILA, University of Colorado, CO 80309-0440, USA.

†Corresponding author.

Electronic address: katori@amo.t.u-tokyo.ac.jp

[1] J. Stenger, H. Schnatz, C. Tamm, and H. R. Telle, Phys. Rev. Lett. 88, 073601 (2002).

[2] M. Niering et al., Phys. Rev. Lett. 84, 5496 (2000).

[3] S. A. Diddams et al., Science 293, 825 (2001).

[4] J. D. Prestage, R. L. Tjoelker, and L. Maleki, Phys. Rev. Lett. 74, 3511 (1995).

[5] R. H. Dicke, Phys. Rev. 89, 472 (1953).

[6] J. C. Bergquist, W. M. Itano, and D. J. Wineland, Phys. Rev. A 36, 428 (1987).

[7] R. J. Rafac, B. C. Young, J. A. Beall, W. M. Itano, D. J. Wineland, and J. C. Bergquist, Phys. Rev. Lett. 85, 2462 (2000).

[8] W. M. Itano, J. C. Bergquist, J. J. Bollinger, J. M. Gilligan, D. J. Heinzen, F. L. Moore, M. G. Raizen, and D. J. Wineland, Phys. Rev. A 47, 3554 (1993).

[9] G. Wilpers, T. Binnewies, C. Degenhardt, U. Sterr, J. Helmcke, and F. Riehle, Phys. Rev. Lett. 89, 230801 (2002).

[10] T. Trebst, T. Binnewies, J. Helmcke, and F. Riehle, IEEE Trans. Instrum. Meas. 50, 535 (2001).

[11] P.S. Jessen and I. H. Deutsch, Advances in Atomic, Molecular, and Optical Physics, edited by B. Bederson and H. Walther (Academic Press, San Diego, 1996), Vol. 37, p. 95.

[12] H. Katori, T. Ido, and M. Kuwata-Gonokami, J. Phys. Soc. Jpn. 68, 2479 (1999).

[13] H. Katori, in Proceedings of the 6th Symposium on Frequency Standards and Metrology (World Scientific Publishing Co., Singapore, 2002), pp. 323-330.

[14] S. E. Hamann, D. L. Haycock, G. Klose, P. H. Pax, I. H. Deutsch, and P.S. Jessen, Phys. Rev. Lett. 80, 4149 (1998).

[15] A. Kaplan, M. F. Andersen, and N. Davidson, Phys. Rev. A 66, 045401 (2002).

[16] C. Cohen-Tannoudji, Fundamental Systems in Quantum Optics in Les Houches, edited by J. Dalibard, J. M. Raimond, and J. Zinn-Justin (North-Holland, Amsterdam, 1992).

[17] T. Ido, Y. Isoya, and H. Katori, Phys. Rev. A 61, 061403(R) (2000).

[18] T. Mukaiyama, H. Katori, T. Ido, Y. Li, and M. KuwataGonokami, Phys. Rev. Lett. 90, 113002 (2003).

[19] H. Katori, T. Ido, Y. Isoya, and M. Kuwata-Gonokami, Phys. Rev. Lett. 82, 1116 (1999).

[20] F. Diedrich, J. C. Bergquist, W. M. Itano, and D. J. Wineland, Phys. Rev. Lett. 62, 403 (1989).

[21] H. G. C. Werij, C. H. Greene, C.E. Theodosiou, and A. Gallagher, Phys. Rev. A 46, 1248 (1992). 